\title{
tic\&société
}

Vol. 5, n²-3 | 2e sem. 2011 / 1er sem. 2012

Les TICs dans les pays des Suds

\section{La gouvernance discutable de l'UIT. Le projet Africa ONE comme exemple}

Jean-Louis Fullsack

\section{OpenEdition}

Journals

Édition électronique

URL : http://journals.openedition.org/ticetsociete/1089

DOI : 10.4000/ticetsociete. 1089

Éditeur

Association ARTIC

Référence électronique

Jean-Louis Fullsack, " La gouvernance discutable de l'UIT. Le projet Africa ONE comme exemple », tic\&société [En ligne], Vol. 5, n²-3 | 2e sem. 2011 / 1er sem. 2012, mis en ligne le 19 juin 2012, consulté le 02 mai 2019. URL : http://journals.openedition.org/ticetsociete/1089 ; DOI : 10.4000/ ticetsociete.1089 
tic\&société - 5 (2-3), $2^{\text {ème }}$ semestre 2011 - $1^{\text {er }}$ semestre 2012

\title{
La gouvernance discutable de I'UIT Le projet Africa ONE comme exemple
}

\author{
Jean-Louis FULLSACK \\ Directeur adjoint honoraire de France Télécom \\ Ancien expert principal et directeur de projets près I'UIT (1978-1998) \\ Administrateur de CSDPTT (www.csdptt.org) \\ Président de CESIR (www.cesir.net) \\ Chercheur associé à la Chaire Unesco, Université de Strasbourg
}

Directeur adjoint honoraire de France Télécom (FT) et ancien Expert principal de l'Union Internationale des Télécommunications (UIT), Jean-Louis FULLSACK est accrédité au Sommet Mondial de la Société de l'Information (SMSI) organisé par les Nations unies. Son domaine d'expertise comprend les technologies, l'ingénierie et l'architecture des réseaux publics de télécommunications, leur économie et leur évolution. Pour le compte de France Télécom il a assuré diverses responsabilités dans la conception, la planification, la mise en œuvre et la maintenance/gestion des réseaux. Pour I'UIT il a été Expert et Coordonnateur de divers projets et actions en Afrique entre 1978 et 1998. II est enseignant vacataire au Groupe des Ecoles de Télécommunications et membre actif de diverses associations professionnelles ou sociales comme l'Institut de Recherches Economiques et Sociales sur les Télécommunications (IREST) et l'International Solar Energy Society (ISES). II est en outre Chercheur associé à la Chaire UNESCO à l'Université de Strasbourg et président fondateur du Centre d'études de la synergie inter-réseaux (CESIR).

II est l'auteur ou co-auteur de divers ouvrages sur les télécommunications et la mise en œuvre des réseaux dans les pays en développement et codirecteur de l'ouvrage "Ethique de la société de l'information" (Editions Bruylant, 2008). 


\title{
La gouvernance discutable de I'UIT Le projet Africa ONE comme exemple
}

\begin{abstract}
Résumé
Le manque d'infrastructures handicape cruellement le développement de l'Afrique. Pour ce qui concerne les télécommunications, des progrès notables ont été enregistrés dans les années 1970-1990 sous l'égide de l'UIT. Mais le dogme néolibéral que l'UIT a endossé dès 1990 a changé complètement le paradigme du développement des réseaux. Le projet Africa ONE en a été une première illustration, et a clairement montré la dérive de I'UIT. Dix ans de sa gestion chaotique du projet ont entraîné un échec lamentable. La conséquence la plus importante a été le gel des autres projets en cours de réseaux satellitaires et terrestres.

L'approche néolibérale que I'UIT a imposé à ses États membres a conduit à une déréglementation à tout va, sans étude préalable d'impact. La «loi du marché " voit les côtes africaines envahies de câbles sous-marins, déployés de manière anarchique et sans vision de réseau. Les réseaux terrestres suivent la même loi : la concurrence fait surgir une multiplicité d'artères sur les segments rentables. Ces investissements inconsidérés engloutissent des milliards d'euros avec le soutien des institutions internationales de financement du « développement».

Pour mettre fin à cette dérive de l'UIT initiée par Africa ONE, l'auteur conclut en appelant à un recentrage des activités et de la stratégie de cette agence onusienne sur ses principes fondamentaux, et notamment à son implication plus efficace dans le déploiement des réseaux dans les pays en développement. Enfin, I'UIT devra s'ouvrir à la société civile, garante de la nécessaire transparence dans sa future gouvernance.
\end{abstract}

Mots-clés : Africa One - UIT - SMSI, gouvernance, télécommunications, Afrique

\begin{abstract}
The lack of infrastructures dramatically handicaps Africa. As far as telecommunications are concerned, noticeable progress was registered during the 1970-1990 decades under the auspices of ITU. But the neoliberal dogma endorsed by the ITU as soon as 1990 has completely changed the networks development paradigm. The Africa ONE Project was a first
\end{abstract}


illustration of this change, and has clearly shown this drift of the ITU. The ten years long chaotic project management ended in a lamentable failure.

The neoliberal approach that the ITU imposed to its Member states lead to an unbridled deregulation, without any preliminary study. The "rule of the market" shows the African coasts flooded with submarine cables, anarchically deployed without any network vision.

Terrestrial links follow the same rule : competition arises a multiplicity of links on profitable segments. These excessive investments squander billions Euros with the support of international "development" constituencies.

For putting an end to this drift of the ITU initiated by Africa ONE, the author concludes by appealing this UN agency to refocusing its activities and strategy on its fundamental principles, in particular being more effectivevely committed in deploying networks in developing countries. At last, the ITU should open itself to the civil society, vouching for the transparency needed in its future governance.

Keywords : Africa One - IUT - WSIS, gouvernance, telecommunications, Africa

\section{Resumen}

La falta de infraestructuras dificulta gravemente a Africa. En lo que concierne las telecommunicaciones, progresos notables hicieron durante los decenios 1970-1990 bajo los auspicios de la UIT. Pero el dogma neoliberal que la UIT asumo desde 1990, completamente cambio el modelo de desarrollo de las redes. El proyecto Africa ONE fue la primera ilustracion de esto cambio, y mostro claramente la deriva de la UIT. Diez anos de su caotica gestion del proyecto han ocasionado su lamentable fracaso. La consecuencia mas importante fue la suspension de otros proyectos de redes en curso, por satélite y terraqueas.

El enfoque neoliberal que la UIT imponio a sus Estados miembros llevo a la desregulacion sistematica, sin ningun estudio previo de impacto. La « ley del mercado " ve las costas africanas invaditas por unos cables submarinos, desplegados de manera anarquica y sin idea de red. Las redes terraqueas siguen la misma ley : la competencia hace surgir una multiplicidad de arterias sobre los segmentos rentables. Estas inversiones desconsideradas tragan unos millares de euros con el sostén de las instituciones internacionales de financiamiento del « desarrollo ». 
Para parar esta deriva de la UIT iniciada por Africa ONE, el autor concluide que esta agencia de la ONU tiene que volver al cientro de sus actividades y de su estrategia, particularmente para implicarse mas eficazmente en el despliegue de los redes en los paises en desarrollo. Al fin, la UIT tiene que abrirse a la sociedad civil, garante de la necesaria transparencia en su futura gobernanza.

Palabras clave: AfricaOne, CMSI, Gobernabilidad, UIT, telecomunicaciones, África. 
La gouvernance discutable de I'UIT : Le projet Africa ONE comme exemple

A la mémoire de mon ami Pierre Mvouama,

Africain de conviction et référence morale, en profonde reconnaissance pour ses apports inestimables dans nos échanges.

Jean-Louis Fullsack

\section{L’Afrique, les réseaux et le développement}

En 2011, l'Afrique est de loin le continent le plus à la traîne du " développement", et la première cause de cette situation, dramatique pour l'avenir de ses populations, est largement imputable à son manque cruel d'infrastructures de base: transport, énergie, eau et assainissement, télécommunications. Les raisons en sont multiples, mais celle qui prévaut est le coût énorme de réalisation de ces infrastructures au niveau national, subrégional et continental. La Banque mondiale (BM) estime à près d'une centaine de milliards de dollars par an sur une dizaine d'années le montant des investissements cumulés nécessaires pour doter le continent d'infrastructures générales répondant à ses véritables besoins.

Le présent article se focalise sur les infrastructures de réseau de télécommunications et des technologies de l'information et de la communication (TIC), sans oublier cependant que les infrastructures - quelle qu'en soit leur nature - sont les bases physiques des réseaux spécifiques qu'elles constituent, réseaux qui délivrent des services qu'attendent les divers acteurs, politiques et économiques, mais aussi les usagers particuliers, à condition qu'ils répondent à des critères essentiels et qu'ils soient gérés, entretenus et développés en fonction des besoins des économies nationales et d'une population fortement croissante et de plus en plus urbanisée.

L'avènement du néolibéralisme et le règne de la "pensée unique " sont, sans conteste, la deuxième raison de l'état déplorable et de l'incohérence des réseaux de télécommunications en Afrique. En effet, ils ont imposé aux États africains, dès le début des années 1990, les plans d'ajustement structurel (PAS) et la déréglementation des secteurs qualifiés de "marchands », dont celui des

${ }^{1}$ Dans le texte qui suit, nous parlerons d'infrastructures ou de réseaux télécoms/TIC . 
télécommunications. La privatisation des offices nationaux des télécommunications et l'ouverture à la concurrence des réseaux ont été menées sous la pression conjointe des institutions financières internationales (Banque mondiale, Fonds monétaire international, Organisation mondiale du commerce, Banque africaine de développement, etc.) et - tout particulièrement - de l'Union internationale des télécommunications (UIT).

\section{L'UIT, un partenaire important pour l'Afrique}

Or, l'une des fonctions qui fonde cette organisation intergouvernementale et agence spécialisée des Nations unies pour les télécommunications ${ }^{2}$ est précisément le déploiement cohérent et harmonieux des télécommunications sur l'ensemble du globe, avec une attentionn spéciale pour les pays en développement $(\mathrm{PeD})$ et donc pour l'Afrique ${ }^{3}$. C'est le rôle exclusif de son Bureau de développement des télécommunications (BDT) ${ }^{4}$. En Afrique, I'UIT a joué un rôle important et croissant à partir de l'accès à l'indépendance des pays anciennement colonisés. C'est ainsi que, dès 1962, et comme en écho au mouvement panafricain renaissant, est née en son sein l'idée d'un réseau panafricain de télécommunications reliant tous les pays du continent: le Panaftel. II a fallu de longues années pour sa gestation, et sa mise en œuvre n'a débuté qu'en 1975. Pendant quinze ans, ce projet, qui a su associer dans une grande aventure technique et humaine les acteurs des pays industrialisés et des pays en développement, a déployé ses artères en Afrique sub-saharienne sous la conduite de I'UIT et avec le concours des institutions nationales et internationales spécialisées dans le développement. C'était l'âge d'or de la Coopération technique et des télécommunications en tant que service public et élément de la souveraineté nationale .

\footnotetext{
2 Voir Chapitre I Article 1 de la Constitution de I'UIT dans Recueil des textes fondamentaux de l'Union internationale des télécommunications adoptés par la Conférence de Plénipotentiaires, édition 2011.

3 Sur les 193 États membres de I'UIT, 43 sont des États de l'Afrique sub-saharienne.

4 Créé par une décision de la Conférence des plénipotentiaires de l'UIT à Nice en 1989, il remplace le Département de la coopération technique en 1991. Son directeur, « ${ }^{\circ} 3$ » de I'UIT, sera Africain jusqu'à aujourd'hui, à l'exception de la période 2006 -2010.

5 Voir Michel Egger et Jean-Louis Fullsack. « Swisscom, l'UIT et la coopération au développement : le néolibéralisme contre la solidarité " Annuaire suisse de politique du développement 2003, Institut universitaire d'études du développement, Genève, p. 95-112.
} 
En 1990, le Panaftel comporte 40000 km d'artères terrestres, dans leur quasi-totalité à base de faisceaux hertziens, et $8000 \mathrm{~km}$ de câbles sous-marins, ainsi qu'une quarantaine de centres de transit international. En mai 1996 à Abidjan, la Conférence régionale africaine de développement des télécommunications organisée par l'UIT (AF-CRDT-96) a donné mandat au BDT pour relancer et achever le réseau Panaftel ${ }^{6}$. Cet objectif se justifiait en particulier par le coût annuel de plus de 400 millions de dollars de l'acheminement du trafic international intra-continental qui, faute de liaisons directes entre États africains, devait transiter par les anciennes métropoles coloniales ou les États-Unis par voie satellitaire. À cette ponction sévère pour les offices publics des télécommunications (OPT) s'ajoutait, à partir des années 1990, la pratique du rappel (call-back), stimulée par la déréglementation ambiante, qui détournait plus ou moins légalement une somme estimée alors à 300 millions de dollars par an au titre des communications internationales. Mais entre temps et à l'instar des organisations du système des Nations unies, I'UIT avait fondamentalement changé de stratégie en devenant l'applicateur zélé de la " pensée unique » imposée par les institutions internationales de financement et du commerce (Banque mondiale, FMI, GATT puis OMC). Elle va tout faire pour "réformer le secteur», entendant par là la déréglementation des télécommunications, et imposer à son tour à ses États membres la privatisation de leur $\mathrm{OP}_{7}^{\top}$ et l'ouverture du "secteur des télécommunications » à la concurrence . La vision panafricaine et la grande aventure de Panaftel ont été dissoutes dans le nouveau dogme néolibéral.

Or, à l'ouverture à la concurrence, les pays africains n'avaient pas achevé leur réseau national, ni - a fortiori - les réseaux sous-régionaux (exemple : le réseau d'interconnexion de la Communauté économique des États d'Afrique de l'Ouest, CEDEAO). Les nouveaux opérateurs privés pouvaient donc rapidement réaliser les segments les plus rentables. En outre, parce que leur réseau était incomplet et souvent très hétérogène, les opérateurs historiques publics voyaient leur patrimoine nettement sous-évalué et devenaient de ce fait des

\footnotetext{
${ }^{6}$ La résolution 4 adoptée par la Conférence précisait dans son paragraphe c) «que le réseau Panaftel doit être pleinement opérationnel en l'an 2000, conformément au Traité d'Abuja portant création de la Commission économique africaine » (CEA).

7 Deux hommes ont marqué cette évolution néolibérale : Pekka Tarjanne, secrétaire général de I'UIT pendant toute la décennie 1990, et Ahmed Laouyane, directeur du BDT de 1991 à 1998.

8 Pour l'Afrique sub-saharienne, des institutions internationales, notamment africaines, distinguent 5 entités politico-géographiques sous l'appellation "sous-région »: l'Afrique de l'Ouest, l'Afrique Centrale, l'Afrique de l'Est, l'Afrique du Sud-est et l'Afrique Australe. Ces régions sont dotées d'organismes institutionnels à "géométrie très variable », car fondés sur des bases diverses, généralement économiques ou géographiques mais aussi d'influences politiques (Afrique Australe).
} 
cibles faciles et bon marché pour les repreneurs, généralement étrangers. L'UIT a ainsi commis une faute lourde de conséquences en faisant de la privatisation du secteur des télécommunications sa stratégie et sa priorité. Ce ne sera hélas ! - pas la seule comme le montrent l'exemple le plus emblématique à cet égard, Africa ONE, et le développement anarchique, incohérent et d'un coût exorbitant des infrastructures des réseaux nationaux africains, de leur interconnexion et de leur insertion dans les réseaux mondiaux. Cette dernière tâche a, en effet, donné naissance à une multiplicité de câbles sous-marins le long des côtes africaines, non seulement sans cohérence entre eux mais aussi largement au-dessus des besoins, même à long terme, du continent. Dans ce qui suit, nous tenterons d'expliquer comment la faillite lamentable du projet Africa ONE a conduit à cette situation à tous points de vue discutable.

\section{Africa ONE : un projet emblématique néolibéral}

\subsection{Une naissance trouble}

En 1993, le directeur du BDT, M.Ahmed Laouyane, prend l'initiative de solliciter AT\&T Submarine Systems International (ATT/SSI) afin de " trouver une voie pour améliorer l'état des communications à travers l'Afrique, en particulier dans la transmission d'État à État »". La réunion a lieu à Hawaï où le directeur du BDT se rend pour reconnaître implicitement l'incompétence de I'UIT dans les fonctions qui constituent la base de ses attributions. Elle est rapportée par deux sources extérieures ${ }^{1}$ et la lecture de leur article montre que, dès sa gestation, le projet Africa ONE s'engage dans un cheminement très particulier. Car ATT/SSI n'est pas la seule société spécialisée dans le secteur du câble sousmarin ; au moins quatre autres sociétés ont alors des compétences reconnues dans ce domaine: Alcatel, Tyco, la filiale de Siemens NSW, et NEC. En outre, un tel projet est non seulement d'une importance stratégique extraordinaire mais aussi de coûts d'étude puis de réalisation impressionnants. Deux caractéristiques qui auraient obligatoirement dû conduire I'UIT à passer par un appel d'offres international ${ }^{\text {. }}$.

${ }^{9}$ Cité par Pat Blake dans «Telecom by sea - The ambitious Africa ONE project promises to be the foundation for teledensity growth on the African continent », Global Telephony, May 1998.

10 Outre l'article de Pat Blake cité, l'événement est rapporté par Hyawatha Bray, « The wiring of a continent - A 1,8 billion ring around Africa», p.A25, Boston Globe, 22/7/2001

${ }^{1}$ Vu son ampleur et son coût présumé, un tel projet devait en outre être entériné auparavant par la Conférence des plénipotentiaires de I'UIT ou, à défaut, être inscrit dans le Plan d'action élaboré par

tic\&société - 5 (2-3), $2^{\text {ème }}$ semestre $2011-1^{\text {er }}$ semestre 2012 
La gouvernance discutable de I'UIT : Le projet Africa ONE comme exemple

En fait, lors de cette réunion à Hawaï, c'est le directeur du BDT de I'UIT, Ahmed Laouyane, qui a attiré l'attention du directeur d'AT\&T Submarine, William Carter, sur l'opportunité commerciale exceptionnelle que présente l'Afrique pour sa société ${ }^{2}$ ! Ce précieux - mais pour le moins paradoxal conseil est bien compris chez AT\&T. Ce sera la société américaine qui concevra, réalisera et exploitera - pendant une durée déterminée - ce grand réseau qui doit relier les pays africains côtiers et intégrer le continent dans le réseau mondial.

En novembre 1995, une "Réunion consultative des membres africains de I'UIT » se tient à Tunis ${ }^{13}$ et les 30 délégations nationales présentes y adoptent une recommandation stipulant que " le BDT sera un participant actif [sic] dans le développement et la mise en œuvre d'Africa ONE, le câble sous-marin qui entourera le continent africain, pour le connecter directement à l'autoroute de l'information " ${ }^{14}$. Le document officiel de I'UIT qui rend compte de cet événement précise plus loin : "Ahmed Laouyane, directeur du BDT et auteur [sic] du concept original d'Africa ONE lancé en 1993 [...] a déclaré que I'UIT (BDT) était préparée à assumer [sa] responsabilité dans le projet Africa ONE ». Le Président d'AT\&T Submarine Systems, William Carter, a déclaré aux délégations africaines que "les travaux de construction d'Africa ONE commenceront en 1996 et seront achevés en 1999. Le coût total sera de 2,6 milliards de dollars ". La seule fausse note viendra du directeur général de Rascom, le projet de satellite africain devant couvrir le continent, qui presse les pays participant à la réunion de "soulever tous les problèmes en rapport avec le projet Africa ONE qui jusque là et regrettablement [siç] n'a vu aucun pays africain impliqué dans aucune de ses différentes étapes " .

Pour I'UIT, la Conférence régionale africaine de développement des télécommunications réunie à Abidjan en mai 1996 (AF-CRDT-96) " a donné une impulsion décisive au projet. Les ministres et les directeurs généraux des télécommunications y ont en effet adopté la Résolution 5 aux termes de laquelle

la Conférence mondiale de développement des télécommunications, en l'occurrence celle de Buenos Aires en mars 1994.

12 Rapporté par Patricia Bagnell, dans l'article cité de Hyawatha Bray dans le Boston Globe.

${ }^{13}$ M. Ahmed Laouyane est Tunisien.

${ }^{14}$ Voir document ITU/95-26 de I'UIT, intitulé ITU to be actively involved in the development of Africa ONE.

15 Selon AT\&T, cet investissement pourrait être amorti en l'espace de cinq à six ans (in : Nouvelles de I'UIT, $\left.\mathrm{n}^{\circ} 1 / 96\right)$

${ }^{16}$ Voir document ITU/95-26 cité 
Africa ONE était reconnu comme projet ayant pour objet de relier tous les pays africains désireux d'y participer. "

\section{2 Africa ONE tangue...}

Premier accroc dans ce projet partagé entre AT\&T Submarine et I'UIT : la Banque mondiale a conditionné sa participation au financement à une réduction de 38 à 20 du nombre de sites d'atterrissement, "ce nombre étant mieux à même de traiter le trafic de télécommunication prévu ». Un pavé dans la mare de I'UIT ! En fait, la raison principale pour la BM était de réduire sensiblement le coût de ce mégaprojet ; il baisse ainsi et sans justification explicite de 2,6 à 1,6 milliard de dollars. Ce faisant, on est passé du concept d'accès pour tous à un concept purement financier.

Deuxième accroc en juillet 1997 : AT\&T Submarine est racheté par Tyco International Ltd. Ce qui remet en cause les promesses initiales. À cette date Africa ONE a fait l'objet de 18 réunions sur le continent et aux États-Unis ${ }^{18}$ qui ont pour but essentiel de mobiliser les pays africains et de leur faire signer le mémorandum d'accord assorti du versement d'un « montant minimal d'un point d'atterrissement [...] maintenu à 15 millions de dollars US »". En outre, I'UIT ajoute une note dans son mensuel officiel qui ne manque pas d'intriguer. En effet, à la fin de l'article sur Africa ONE et sous le titre SAT-3 on peut lire : " $\mathrm{Ce}$ système acheminera le trafic entre l'Afrique du Sud et l'Europe, lorsque la capacité du SAT-2 en place actuellement sera saturée. Etant donné qu'Africa ONE reliera aussi l'Afrique du Sud à l'Europe, il sera inutile de prévoir un câble séparé pour SAT-3 [sic]. Tout est mis en œuvre actuellement pour assurer l'intégration de ce câble ». On verra que la réalité fera un joli pied de nez à cette affirmation péremptoire de l'UIT.

À la suite du rachat d'AT\&T/SSI, les dirigeants de Tyco se trouvent impliqués dans un gigantesque scandale financier comme le révèlera plus tard l'enquête de la Commission de sécurité de la Bourse américaine (SEC). Si les liens du détournement de 600 millions de dollars avec cette acquisition restent à démontrer, ils sont indéniablement une cause du gel relatif pendant deux ans du

\footnotetext{
${ }^{17}$ Voir le Rapport de la Conférence AF-CRDT-96 à www.itu.int/itudoc/itu-d/wtdc98/docs/03-fr.pdf 18

Dont trois réunions des coordonnateurs nationaux de 28 pays ayant signé le mémorandum d'accord avec AT\&T/SSI. Le président du comité de coordination est M. Laouyane, directeur du BDT.

${ }^{19}$ Voir Africa ONE : messages encourageants des coordonnateurs nationaux in Nouvelles de l'UIT, $3 / 97$.
}

tic\&société - 5 (2-3), $2^{\text {ème }}$ semestre $2011-1^{\text {er }}$ semestre 2012 
projet Africa ONE. Il a fallu une nouvelle cession d'Africa ONE Ltd à Columbia Technologies (New Jersey) ${ }^{20}$, puis la réunion des directeurs généraux des télécommunications africains convoqués en Tunisie en 1999 par l'UIT, pour le relancer. Entre temps, I'UIT met Africa ONE à l'ordre du jour de sa Conférence mondiale de développement des télécommunications, réunie à La Valette (Malte) en mars 1998, sans informer ses membres des difficultés importantes rencontrées suite à ces péripéties, notamment au niveau du financement ${ }^{21}$. Conséquence directe de cette gestion chaotique du projet Africa ONE, son géniteur, M. Ahmed Laouyane, n'est pas réélu lors de la Conférence des plénipotentiaires de Minneapolis en octobre 1998 (PP-98), les membres de I'UIT lui ayant préféré le malien Hamadoun Touré.

\section{3 Africa ONE ramené à la surface}

C'est la fin d'un épisode, mais l'UIT maintient le projet dans le plan d'action approuvé par la PP-98. La "bulle Internet», qui éclate fin 1999, modifiera sensiblement le paysage autour d'Africa ONE mais ne changera en rien la gestion calamiteuse de I'UIT, toute absorbée par sa promotion de la déréglementation et la privatisation des opérateurs publics africains. Dans cette application sur le terrain de la "pensée unique " néolibérale, la Commission économique pour l'Afrique de l'ONU n'est pas en reste dont le représentant affirme : "Même les pays qui s'agrippaient encore au vieux paradigme [sic] de monopole sont forcés de suivre le pas par des utilisateurs instruits [sic] qui exigent de meilleurs services, et par une pression accrue de la part de la Banque mondiale et de financiers bilatéraux ${ }^{22}$ ». On est alors en pleine illusion d'une "nouvelle ère numérique " qui nourrira les plus grands scandales financiers que le monde ait connus jusque-là, tous dans le domaine des télécommunications $^{23}$, celui que I'UIT est censé piloter en terme de

${ }^{20}$ Cette société est organisée selon la législation du Libéria [sic] avec une agence opérationnelle à New York City. Curieusement, le communiqué de presse de Lucent qui annonce cet événement est édité à New York City, Murray Hill, à New Jersey... et à Hamilton, Bermudes. Voir www.lucent.com/press/0699/990604.coa.html.

21 Africa ONE fait l'objet du point de l'ordre du jour 3.3 de la PP-98, le document 15-E (ou 15-F en français), traduisant la position officielle de I'UIT. On notera que l'Afrique du Sud ne figure pas parmi les signataires du Mémorandum d'accord annexé à ce document.

22 Financial Times, 12 octobre 1999.

23 Parmi les plus retentissants, ceux d'Enron, qui est aussi un nouvel opérateur de télécommunications aux États-Unis, et de WorldCom dont les dirigeants étaient reçus 
Jean-Louis FULLSACK

développement cohérent, tout comme en terme de normalisation. Curieusement, cette agence onusienne semble autiste et incapable de percevoir l'ampleur de la crise. Alors que les premiers avertissements quant à la viabilité du projet Africa ONE apparaissent dans les magazines spécialisés ${ }^{24}$, le secrétaire général de I'UIT est convié comme keynote speaker au Sommet de haut niveau [sic] des télécommunications, réuni en mars 2001 à Accra (Ghana). Africa ONE est présenté comme la solution définitive pour l'Afrique par son nouveau mentor, Columbia Technologies ${ }^{25}$, qui a choisi Global Crossing comme réalisateur et gestionnaire du système sous-marin ${ }^{26}$. Mais les pays enclavés se sentent floués par les promesses antérieures de I'UIT, car aucune ne s'est concrétisée quant à leurs artères de télécommunications jusqu'aux stations d'atterrissement d'Africa ONE.

\section{4 Le naufrage annoncé}

Ayant évalué le projet sur le plan technologique, fonctionnel et économique, j'étais dès le début du projet sceptique sur son adéquation et critique quant à la conception de sa mise en œuvre. En effet, sur le plan technologique et opérationnel se posait surtout le problème d'un système très lourd et à la dimension continentale qui n'avait d'équivalent nulle part au monde. Sa gestion était dès lors plus que problématique. Alors que la solution tacitement adoptée jusqu'à l'avènement d'Africa ONE reposait sur les réseaux sous-régionaux disposant chacun de sa capacité de gestion, le réseau continental consistait à relier entre eux chaque réseau sous-régional, facilitant ainsi l'exploitation, la gestion et le développement de l'ensemble. On ne peut pas expliquer rationnellement l'énorme erreur de I'UIT dans ses options et son engagement aveugle en faveur d'Africa ONE, alors qu'elle disposait d'une information technologique du plus haut niveau avec ses groupes d'études de I'UIT-T et de I'UIT-R. II faut aussi ajouter à ce constat le silence des États membres africains de I'UIT, et tout particulièrement les États membres de son Conseil, même s'ils

solennellement à I'UIT, s'exprimaient dans ses conférences et exerçaient leurs talents dans les " groupes de réflexion » pour « réformer » I'UIT.

24 Voir Reuben Muoka "African cable project leaves some stranded», Communications International, April 16th, 2001.

25

Voir la présentation de Glenda Jones www.africaone.com/english/news/NewsDetail.cfm?NID=2001

${ }^{26}$ En janvier 2002, Global Crossing se déclare en faillite (relevée par The Wall Street Journal online, January 29, 2002), après que la société eût investi 13 milliards de dollars dans son réseau sousmarin mondial. Article consultable à www.happinessonline.org//nfectiousGreed/p15.htm

tic\&société - 5 (2-3), $2^{\text {ème }}$ semestre $2011-1^{\text {er }}$ semestre 2012 
ne sont guère des spécialistes de réseau, sachant que le modèle continental qui était la caractéristique d'Africa ONE était contraire à la politique de l'Union africaine - et de son NEPAD - qui basait toute sa stratégie sur les sous-régions, véritables entités dont la fédération était la base politique pour le continent.

Mon grief essentiel, cependant, était d'ordre éthique et concernait les conditions particulières qui ont abouti à confier de gré à gré et dans la plus grande opacité un marché d'étude pour un projet d'une telle importance et estimé à plus de deux milliards de dollars.

Dans un mémorandum, adressé en juillet 1998 et sur sa demande au président de la Commission du développement du Parlement européen (PE), traitant du financement des infrastructures en Afrique par des programmes européens tels que le Fonds européen de développement (FED), j'ai argumenté en faveur d'un moratoire pour le projet Africa ONE. Cette option a retenu l'attention de mon interlocuteur, concrétisée par un entretien particulier consacré à cette problématique. De même, je m'en étais ouvert au BDT de I'UIT dans le cadre de deux missions que j'ai eu à effectuer en Afrique pour son compte, mais sans obtenir de suite. L'association CSDPTT dont je suis alors membre, est la première à évoquer ce projet sur son site et dans sa Lettre mensuelle, à l'évaluer sur le plan technologique et à demander un moratoire, afin de statuer quant à sa faisabilité et surtout de remettre à plat l'étude technique complètement dépassée et trop coûteuse.

Ce sera finalement le Sommet mondial de la société de l'information (SMSI), dont les Nations unies ont confié la coordination à I'UIT en 2001 et qui tient en juillet 2002 sa première réunion préparatoire (PrepCom, dans le jargon adopté), qui fournira le cadre de la contestation du projet Africa ONE. Dans ses interventions au nom de la société civile en réunions plénières intergouvernementales dès le deuxième PrepCom, CSDPTT demande l'arrêt du projet Africa ONE, et le report des sommes qui lui sont allouées à des projets entrant dans le cadre d'un concept de réseau d'interconnexion continental. Car un autre projet de desserte par câble sous-marin des pays africains, riverains de l'Atlantique, dénommé SAT-3/WASC (voir plus loin) et beaucoup plus viable qu'Africa ONE, est annoncé dans la presse spécialisée. En parallèle, CSDPTT demande la priorité pour le projet de desserte satellitaire du continent africain, Rascom, au programme de l'UIT depuis le début des années 1980 mais occulté par les avatars et compromissions de I'UIT dans Africa ONE. 
Jean-Louis FULLSACK

\subsection{Un partenariat public-privé}

La seule constante observée tout au long de la gestion du projet Africa ONE concerne son statut. En 1993-94, c'est une innovation. En effet, ce ne sont plus seulement des opérateurs publics qui conçoivent en commun et co-financent un projet pour l'exploiter ensuite en fonction des contributions de chacun et des attributions convenues en commun, mais une société ou consortium de statut privé (voir au-dessus) qui élabore et réalise ce projet au profit d'une entité créée pour l'occasion - Africa ONE Ltd - et qui cherche son financement auprès d'investisseurs publics et privés. Pour les premiers, c'est l'UIT qui assume le rôle de collecteur, auprès des États membres africains en particulier, mais aussi auprès des institutions financières internationales (Banque mondiale, Banque africaine du développement, etc.). Quant aux investisseurs privés, ce seront essentiellement des investissements-risques qui ont déjà "fait leurs preuves " dans des projets tels que Global Crossing et Oxygen, les futures grandes victimes de la " bulle Internet ${ }^{27}$. Le principe de ce véritable partenariat publicprivé, promu par I'UIT et quelques uns de ses membres de secteur avec la double onction de la Chambre du commerce international $(\mathrm{CCl})$ et du Secrétariat général des Nations unies, repose sur un nouveau concept : BuildOperate-Transfer $(B O T)$. Une fois le projet réalisé, il sera exploité par la société Africa ONE pendant douze ans, puis il sera transféré aux opérateurs nationaux et internationaux qui seront alors tous privatisés.

Ce montage, qui fera quelques adeptes et autant de faillites, a les faveurs des institutions internationales de financement, Banque mondiale en tête qui intervient alors comme garant auprès des bailleurs privés, tout en demandant aux États de "créer des conditions réglementaires favorables" aux investisseurs privés, pour permettre le rapatriement de l'essentiel de leurs bénéfices... dans les paradis fiscaux. Quant au transfert du réseau aux opérateurs, il interviendra au bout d'un délai de 12 ans, suffisamment long pour entraîner l'éclatement des structures des télécommunications dans les pays concernés. D'autre part, cette durée est à la limite de la durée de vie des équipements qui représentent une partie importante de l'investissement et entraînera ipso facto leur renouvellement à la charge des opérateurs... ou de leurs repreneurs. II est pour le moins regrettable que I'UIT n'ait pas mieux évalué ces risques - et cela dès l'origine du projet - dont ses États membres seraient les premières victimes.

27

Naïveté coupable ou ignorance des réalités du monde financier? L'UIT affirme dans son « Journal officiel » : " Grâce à la structure d'Africa ONE, le projet peut-être financé par des capitaux privés afin de combler le déficit, ce qui constitue un filet de sécurité pour l'exécution du projet. » (Nouvelles de l'UIT, $n^{\circ} 7 / 97$, page 34)

tic\&société - $5(2-3), 2^{\text {ème }}$ semestre $2011-1^{\text {er }}$ semestre 2012 
La gouvernance discutable de I'UIT : Le projet Africa ONE comme exemple

\subsection{Africa ONE sombre}

L'éclatement de la « «bulle Internet » - en fait une bulle qui touche tout le secteur des télécommunications - a raison du dernier des gestionnaires successifs du projet Africa ONE, Global Crossing, en charge de sa mise en œuvre. En même temps, I'UIT est en pleine crise financière et affaiblie. Son BDT est de plus en plus discret pour soutenir Africa ONE avec sa capacité de $60 \mathrm{Gbit} / \mathrm{s}$, et regarde vers d'autres projets, plus actuels et mieux encadrés, tels que le câble sous-marin South Africa Telecommunications $n^{\circ} 3 /$ West African Submarine Cable (SAT-3/WASC) dont la capacité initiale annoncée est de 120 Gbit/s.

La faible implication des organisations panafricaines, comme l'Union panafricaine des télécommunications (UPAT, future UAT), et le soutien fléchissant des États africains qui sont plus soucieux de s'interconnecter entre eux et dont les opérateurs nationaux se débattent dans les turbulences de la privatisation imposée, font progressivement disparaître Africa ONE des préoccupations africaines. Mieux, les opérateurs africains se rallient progressivement au modèle plus conventionnel de SAT-3 qui leur garantit une place de co-gestionnaire et leur assure l'ouverture vers le réseau mondial. Tous ces éléments ont rendu inévitable le naufrage d'Africa ONE. Celui-ci a eu lieu dans un grand silence informationnel ${ }^{28}$ auquel I'UIT a largement contribué, et pour cause!

Le projet, officiellement porté par I'UIT dès 1994 comme on l'a vu, allait atteindre sa dixième année lorsqu'il est déclaré " mort né » par le directeur du BDT de l'UIT à la veille du Sommet mondial de la société de l'information de Genève en début de décembre 2003. II a fallu un événement de hasard pour sortir l'UIT de ce silence inadmissible pour les pays et les utilisateurs africains. On s'étonnera aussi du silence des responsables politiques africains, et plus particulièrement de l'absence de réactions des membres africains du Conseil de I'UIT. Le hasard a voulu que le directeur du BDT, M. Hamadoun Touré, accorde un entretien à Michel Egger, coordonnateur des ONG suisses au SMSI et

\footnotetext{
28 Le site d'Africa ONE a vu sa dernière mise à jour le 23 avril 2001 pour rendre compte de la réunion du "Comité consultatif de l'EximBank pour l'Afrique sub-saharienne " du 18 avril à Washington. Celle-ci soutient Africa ONE pour son potentiel important pour les sociétés américaines du secteur des télécommunications. Sa lecture est révélatrice à plus d'un titre. Voir www.africaone.com/french/news/NewsDetail.cfm?NID=2002. De son côté, Lightwave Europe évoque les grands projets de câbles sous-marins dans le monde dans son édition mensuelle d'octobre 2002, sans aucune mention d'Africa ONE.
} 
enseignant à l'Institut universitaire d'études du développement de Genève. Parmi d'autres questions posées figurait celle sur la gestation douteuse d'Africa ONE et l'incertitude sur son sort. En fait, cette question avait été posée par CSDPTT au directeur du BDT deux mois auparavant, lors d'une session du PrepCom du SMSI consacrée au rôle de I'UIT pour combler le "fossé numérique » dans les $\mathrm{PeD}$ et plus particulièrement en Afrique. Cette question, assortie de la proposition d'un moratoire pour le projet Africa ONE, avait visiblement irrité le directeur du BDT qui ne lui a pas donné de réponse. Contacté par Michel Egger pour préparer cet entretien, CSDPTT lui a donc suggéré de la re-poser, ce qui fut fait. La réponse de $M$. Hamadoun Touré vaut d'être intégralement citée ${ }^{29}$ : "C'était avant mon arrivée, et vous comprendrez qu'il m'est difficile de m'exprimer sur ce sujet. Je n'ai pas à juger la politique de mon prédécesseur. Tout ce que je puis vous dire, c'est qu'effectivement les choses ne se sont pas passées comme elles auraient dû [sic] ... ». À la question de Michel Egger "Peut-on estimer que c'est un projet mort-né ? ", le directeur du BDT répond en trois mots : "On le peut ".

Cette réponse lapidaire d'un haut responsable de I'UIT est aussi discutable que difficilement recevable. En effet, elle réduit cette longue et chaotique histoire à un simple problème de personne, éludant ainsi toute la responsabilité de I'UIT. En même temps, il passe sous silence qu'il est depuis cinq ans le successeur de M. Laouyane au BDT et, qu'en tant que tel, il est aussi le président du Comité de coordination d'Africa ONE qui en a conduit toutes les réunions officielles ; il porte donc une part importante de responsabilité dans cette "mort" aussi singulière que discrète, et dans ses conséquences insidieusement passées sous silence. Le directeur du BDT occulte ainsi sa responsabilité personnelle mais implicitement aussi celle de I'UIT.

On ne saura donc rien sur le sort des sommes recueillies par Africa ONE Ltd auprès ${ }_{1}$ des États africains en contrepartie de leur droit d'accès au câble sousmarin $^{31}$, ni sur les montants importants que I'UIT a consacrés à ce projet

\footnotetext{
${ }^{29}$ Voir l'article « Plus que la fracture, c'est l'opportunité numérique qui importe » de M. Hamadoun Touré, propos recueillis par Michel Egger, pp. 113 à 122 dans Annuaire suisse de politique de développement 2003, édité par l'Institut universitaire d'études du développement, Genève.

${ }^{30}$ Page 117, op.cit.

31 À raison de 15 millions de dollars par « droit d'accès » et d'une trentaine d'États ayant signé la convention avec Africa ONE, on peut se faire une idée de l'importance de la question posée aux responsables de I'UIT et en particulier au président du Comité de coordination, même si on admet qu'une partie seulement des sommes dues a été versée.
}

tic\&société - 5 (2-3), $2^{\text {ème }}$ semestre $2011-1^{\text {er }}$ semestre 2012 
controversé pendant près de dix ans. La « tour ${ }^{32}$ de la place des Nations n'est décidément pas un immeuble de verre...

\section{Africa ONE, un révélateur de la dérive de l'UIT}

Ce que ce grave échec de I'UIT révèle d'abord, c'est sa dérive inexorable vers des concepts et des pratiques basés sur l'idéologie néolibérale et le règne de la loi du marché. Cette dérive est éthiquement peu compatible avec les fonctions quj lui ont été attribuées à l'origine, voire avec sa constitution et sa convention $^{33}$. II faudra donc « réformer » et " compléter », article par article, ces textes fondamentaux et cela, dès la conférence de plénipotentiaires de 1990. Cette «plénipo » a eu deux résultats très différents, voire paradoxaux. D'une part, elle a valorisé la contribution de I'UIT au développement en promouvant son département de la coopération technique en bureau du développement des télécommunications (BDT, ou Sęcteur UIT-D), à l'égal des deux autres Bureaux ou Secteurs qui constituent I'UIT ${ }^{34}$. D'autre part, elle a élu le candidat très libéral Pekka Tarjanne ${ }^{35}$ comme secrétaire général de la vénérable institution et ouvert ainsi la voie à une "nouvelle UIT». Le bouleversement 36 est tel qu'il faut organiser une conférence des plénipotentiaires additionnelle ${ }^{36}$ pour faire entrer son changement de cours dans ses textes fondamentaux et adopter cette nouvelle organisation, une première dans la très longue histoire de I'UIT !

Cette «réorientation» de I'UIT, appuyée par la «pensée unique » qui prévaut dans les institutions financières internationales (Groupe de la Banque mondiale, FMI, FED, BAD) qui ${ }_{3}$ sont aussi des partenaires de I'UIT dans le financement des grands projets ${ }^{3}$, offre aussi un terrain idéal pour se lancer dans une aventure telle qu'Africa ONE. Mais la longue et discutable gestation

\footnotetext{
32 Le siège de I'UIT à Genève est un immeuble de 15 étages, dont le $14^{\mathrm{e}}$ est celui du secrétaire général et de son état-major.

33 Voir Projet de recherche CRSH 2005-2008 - Gouvernance mondiale: démocratisation ou privatisation du système international ? Cahier de recherche MCD-UIT-02, de I'Université du Québec à Montréal. URL : www.ieim.uqam.ca/IMG/pdf/2eme_cahier_de_recherche_UIT.pdf .

34

Le bureau de la normalisation ou secteur UIT-T et bureau des radiocommunications ou secteur UIT-R.

${ }^{35}$ Voir Michel Egger et Jean-Louis Fullsack. Swisscom, I'UIT et la coopération au développement : le néolibéralisme contre la solidarité, op.cit.

${ }^{36}$ Elle aura lieu à Genève du 7 au 22 décembre 1992.

${ }^{37}$ Ces projets concourent au budget de I'UIT qui prélève un pourcentage appréciable du montant des projets qu'elle gère. Ces revenus génèrent près de $15 \%$ de son budget.
} 
Jean-Louis FULLSACK

d'Africa ONE a mobilisé les ressources maigres de I'UIT en personnel qualifié, voire en ressources financières, aux dépens de ses autres priorités qu'étaient alors le projet Rascom, le satellite pour l'Afrique, et, en second, le réseau Panaftel.

\subsection{Rascom, première victime}

Rascom est en fait un projet bien antérieur puisque son étude de faisabilité a été financée par le Fonds européen de développement (FED)... en 1980 ! Vingt ans et plusieurs épisodes plus tard, le satellite est encore à terre, alors que les États africains rappellent régulièrement leurs besoins en liaisons satellitaires intra-continentales, ne serait-ce que pour éviter de passer par le système satellitaire européen et nord-américain Intelsat pour assurer le transit des communications d'un pays africain vers un autre, et économiser ainsi les 400 millions de dollars annuellement encaissés par les opérateurs européens et américains ${ }^{38}$. En fait, ce transit, à l'extérieur du continent, du trafic intra-africain traduit l'absence d'un véritable réseau terrestre africain interconnectant l'ensemble des pays via les réseaux des sous-régions telles que l'Afrique de l'Ouest, l'Afrique Centrale, etc. Pallier cette carence - en attendant la réalisation complète du réseau Panaftel - et desservir les vastes zones isolées à travers le continent africain étaient les deux fonctions essentielles de Rascom. Lorsqu'il sera enfin lancé en 2008, son exploitation est compromise par un dysfonctionnement $^{39}$. C'est seulement à partir d'août 2010, avec le lancement du second satellite, que le système satellitaire peut être assuré conformément à son cahier des charges. Mais, à ce moment, le visage des télécommunications a profondément changé en Afrique avec l'essor des câbles sous-marins et des liaisons terrestres à fibres optiques, et surtout avec l'avènement incontestable des communications mobiles qui commencent progressivement à couvrir les zones "rurales ». Ce qui a entraîné une modification des fonctions de Rascom : soutien du réseau électronique panafricain de télé-santé et de téléenseignement, services de téléphonie et Internet aux zones isolées (TES), réception de radio/TV sur tout le continent. De fait, l'objet initial, à savoir assurer l'interconnexion entre pays, n'est plus sa priorité.

38 Alors que ce problème important et récurrent était utilisé comme argument de poids pour justifier le satellite africain dans son étude de faisabilité, il a été détourné ensuite pour justifier la faisabilité... d'Africa ONE. Un double emploi discutable !

39

Voir « Le lancement réussi de Rascom, le premier satellite de communications africain, est remis en cause par une panne mécanique », Balancing Act, édition en français, $n^{\circ} 74$, janvier 2008. URL : www.balancingact-africa.com/node/16345 .

tic\&société - 5 (2-3), $2^{\text {ème }}$ semestre $2011-1^{\text {er }}$ semestre 2012 


\subsection{Panaftel, deuxième victime}

En effet, comme vu plus haut, c'est l'objectif ambitieux fixé au Panaftel, le réseau pan-africain de télécommunications, que I'UIT élabore et met en œuvre progressivement pendant les "vingt glorieuses » de la coopération technique, soit la période $1970-1990^{40}$, grâce à la générosité de bailleurs de fonds internationaux très variés comme la Banque mondiale, le Programme des nations unies pour le développement (PNUD), le Fonds européen de développement (FED), la Banque arabe de développement économique de l'Afrique (BADEA), mais aussi des dons et subventions de pays industrialisés tels que la Caisse centrale de coopération économique (CCCE), la devancière de l'Agence française de développement (AFD), et l'Agence canadienne de développement international (ACDI). Cette dernière a joué un rôle pionnier et on lui doit l'artère hertzienne Dakar-Bamako-Ouagadougou-Niamey, véritable trait d'union des pays sub-sahéliens.

Initialement promis pour l'an 2000 (cf. chapitre 2), I'UIT s'était engagée à te réaliser Panaftel pour 2004 ; il a été abandonné dans le contexte du SMSI sans aucune explication ni information circonstanciée, I'UIT préparant déjà une autre initiative (voir § 7.2). Une victime de plus de la relation symbiotique entre l'UIT et la doctrine néolibérale.

\section{L’UIT, vecteur de la pensée néolibérale}

\subsection{Le tournant néolibéral de I'UIT}

Débuté comme vu plus haut lors de la "plénipo » de Nice en 1989 avec l'élection de Pekka Tarjanne ${ }^{41}$ comme secrétaire général, ce tournant de I'UIT

\footnotetext{
40 Voir Jean-Louis Fullsack. «La coopération multilatérale dans le secteur des télécommunications ", in: Société numérique et développement en Afrique, sous la direction de Jean-Jacques Gabas, GEMDEV-Karthala, 2004, p. 331-357

41

Ancien ministre finlandais des Transports et Communications et directeur général des postes et télécommunications, il a été élu secrétaire général de I'UIT lors de la Plénipo'89 à Nice et l'est resté dix ans. Quelques semaines après avoir quitté la direction de I'UIT, il a été embauché - sans états d'âme quant à l'éthique - comme " $\mathrm{n}^{\circ} 2$ » du projet Oxygen, concurrent de Global Crossing dans les réseaux sous-marins mondiaux! Voir son curriculum à www.itu.int/net/itunews/issues/2010/03/04.aspx, particulièrement révélateur quant à l'orientation qu'il a imprimée à l'UIT.
} 
vers les thèses néolibérales les plus orthodoxes prône une transition qui casse le modèle historique sur lequel elle s'est fondée ${ }^{42}$. À peine élu, le nouveau secrétaire général se prévaut d'avoir été le premier en Europe à avoir mis en œuvre la déréglementation des télécommunications, et impose son idée de " nouvelle UIT » avec un rôle renforcé de ses membres du secteur privé. Le tournant néolibéral atteint un nouveau degré à la "plénipo " de Marrakech en 2002 . Sur le plan politique, il se traduit par une incitation forte - car conditionnée par l'assistance et le financement des programmes d'investissements - à la déréglementation des télécommunications publiques assurées jusque-là par les Offices publics des télécommunications (OPT) en Afrique francophone. Ce choix, délibéré et dogmatique en faveur de la déréglementation, est imposé à l'ensemble des $\mathrm{PeD}$ jusqu'aux plus pauvres d'entre eux, les PMA. En le généralisant sans discernement ni étude préalable d'impact socio-économique et structurel, I'UIT commet non seulement une erreur grave de conséquences mais elle trahit aussi la confiance de ses États membres africains dont elle est censée représenter et défendre les intérêts ${ }^{4}$. Jusque-là grands services publics, les télécommunications vont devenir progressivement des services purement marchands assurés par des sociétés privées qui se concurrencent mutuellement et qui cherchent le meilleur rendement de leur investissement.

La transformation du Département de la coopération technique en Bureau du développement (BDT) n'est pas seulement structurelle mais aussi politique. Dès lors, I'UIT est davantage attachée à soutenir les initiatives privées dans le sens voulu par ses "membres du secteur" - constructeurs, vendeurs et opérateurs pour la plupart - qu'à susciter et faciliter des initiatives de ses États membres. Ce qu'a fort bien documenté l'affaire Africa ONE et ce que continuent actuellement à démontrer d'autres erreurs commises dans les projets de réseaux terrestres et dans le déploiement anarchique des câbles sous-marins le long des côtes africaines comme le montre le chapitre 6.

\footnotetext{
42 Voir l'article « L'UIT : l'agence spécialisée des Nations unies, acteur déterminant dans l'évolution néolibérale du secteur des télécommunications », www.csdptt.org/article424.html.

43 Voir projet de recherche CRSH 2005-2008 - Gouvernance mondiale : démocratisation ou privatisation du système international ? op.cit.

44 Voir Jean-Louis Fullsack. " L'UIT, acteur déterminant dans l'évolution libérale du secteur des télécommunications », op.cit.
} 
La gouvernance discutable de l'UIT : Le projet Africa ONE comme exemple

\subsection{La ligne conservatrice de I'UIT}

Alors que, progressivement, les agences et programmes des Nations unies s'ouvrent à la société civile, l'UIT sait résister à cette évolution qu'elle juge comme une menace pour sa stabilité. Elle veille au contraire à son partenariat, particulier et exclusif, avec le secteur privé qu'elle a fait entériner dans sa Constitution et sa Convention. En outre, l'assemblée générale des Nations unies a décidé la nature tripartite du SMSI : gouvernements, secteur privé, société civile, et a désigné I'UIT comme organisatrice et coordinatrice de ce sommet onusien. Cette situation nouvelle aurait dû inciter l'UIT à s'ouvrir - aussi - à la société civile, comme l'a rappelé la question de Michel Egger à Hamadoun Touré, alors directeur du BDT ${ }^{45}$. Tout en déclarant que les responsables de I'UIT "[sont)] ouverts aux ONG, tout à fait disposés à travailler avec elles " mais en ajoutant aussitôt : " $\dot{A}$ notre avis, elles n'ont pas besoin de devenir formellement membres de secteur pour cela. Ce que nous refusons [sic], c'est une politisation de l'UIT. Nous faisons du développement, pas de politique ". Ainsi, pour le futur secrétaire général, la participation active des entreprises du secteur privé aux choix stratégiques de I'UIT et à la promotion de la pensée unique néolibérale, n'a aucun caractère politique, pas plus que la déréglementation et la privatisation des Offices publics des télécommunications imposées à marche forcée aux PeD !

Cette question relayait les propositions récurrentes de CSDPTT tout au long du SMSI, puis ultérieurement dans son processus de suivi, pour une ouverture de I'UIT aux organisations de la société civile, spécialisées dans l'action et la réflexion dans le domaine des TIC pour le développement. Les propositions stipulaient que ces ONG seraient admises comme membres du Secteur du développement, à parité de droits avec le secteur privé, et dispensées de versement de cotisation en échange de leur coopération active avec le BDT. Entre temps, les conférences des plénipotentiaires d'Antalya (2006, Turquie) et de Guadalajara (2010, Mexique) ont abouti à la création d'un nouveau type de membres de I'UIT : les membres associés. Les membres associés du Secteur du développement paient une cotisation annuelle de 3975 Francs suisses (la moitié pour les membres associés des $\mathrm{PeD}$ ) et ont des rôles limités à des projets ${ }^{46}$, conditions dissuasives pour la grande majorité des ONG intéressées.

\footnotetext{
45 Voir Hamadoun Touré. « Plus que la fracture, c'est l'opportunité qui importe », p. 120, op.cit.

46 Contrairement aux membres des secteurs (Normalisation, Radiocommunications, Développement) du secteur privé qui paient une cotisation de 7950 Francs suisses (la moitié pour ceux des PeD), les membres associés n'ont pas accès aux groupes consultatifs qui contribuent à la définition des politiques de l'UIT dans ses trois secteurs.
} 
L'UIT se préserve ainsi non seulement des "risques de politisation " évoqués par Hamadoun Touré, mais aussi de tout obstacle éventuel dans sa trajectoire néolibérale.

\section{6 Évolution des réseaux en Afrique et loi du marché}

\subsection{Réseaux terrestres}

En absence de tout schéma directeur ou de concept intégrateur tel que le réseau Panaftel, les projets et réalisations d'artères structurantes à fibres optiques se multiplient sur le continent, conformément à la loi du marché et avec la tacite complicité de I'UIT. Les projets tels que celui du Central African Backbone ( $\mathrm{CAB}$ ) ou les mises en œuvre le plus souvent problématiques d'artères à fibres optiques au Kenya, en Ouganda et République démocratique du Congo pour ne citer qu'elles, illustrent cette autre dérive de I'UIT et de son BDT, mais aussi l'inefficacité de son bureau régional Afrique et de ses quatre bureaux de zone ${ }^{4}$. Non seulement ces artères sont réalisées au prix fort et sans aucune cohérence avec des projets ou réalisations concurrents, mais elles échappent pour une grande partie à tout concept de réseau et constituent pour certaines d'entre elles des doublons inqualifiables. Dans d'autres cas, on voit des câbles à fibres optiques réalisés le long d'une route qui vient d'être construite, ou qui fait l'objet d'un projet confirmé, alors que l'intégration du projet de câble dans le projet routier aurait éçonomisé jusqu'à une dizaine de milliers de dollars par kilomètre de câble posé ${ }^{48}$ !

\section{CAB : Un projet très discutable}

Le cas du projet CAB (Central African Backbone, ou réseau dorsal d'Afrique Centrale) illustre bien toutes ces carences et met en outre en évidence le manque de compétence du BDT de I'UIT dans le domaine technologique et d'architecture de réseau.

47 Créé en 1992, le Bureau régional Afrique a son siège à Addis Ababa (Éthiopie); ses bureaux de zone sont à Yaoundé (zone Afrique centrale), Addis Abeba (zone Afrique de l'est), Harare (zone Afrique du sud) et Dakar (zone Afrique de l'Ouest). Cette représentation régionale dépend du BDT.

${ }^{48}$ C'est un des nombreux effets bénéfiques de la synergie inter-réseaux (SIR) que défend le CESIR auprès des grandes institutions d'investissement (Banque mondiale, Banque africaine du développement, BAD, Banque européenne d'investissement, BEI) et d'acteurs du développement (Commission européenne, Parlement européen, Pays ACP, UIT, etc.).

tic\&société - 5 (2-3), $2^{\text {ème }}$ semestre $2011-1^{\text {er }}$ semestre 2012 
En effet, sur financement de la Banque mondiale, l'UIT avait été chargée de l'étude de faisabilité de ce réseau dorsal pour l'Afrique centrale. Ignorant les projets - dont certains très avancés - d'artères nationales à fibres optiques dans la sous-région ${ }^{49}$, l'équipe de I'UIT s'est appuyée sur ses propres hypothèses en basant son étude sur l'utilisation de l'artère à fibres optiques posée le long de l'oléoduc entre le Tchad et le Cameroun. En conséquence, la topologie du réseau qu'elle propose s'avère inadéquate pour un réseau de cette importance.

Clause aggravante: l'étude de I'UIT n'a pas pris en compte les projets d'infrastructure en cours dans la sous-région tels que le corridor routier $n^{\circ} 1$ Yaoundé-Brazzaville et le corridor routier $n^{\circ} 2$ Yaoundé-Bangui, soutenus par le NEPAD au titre de l'intégration sous-régionale, ni le projet d'interconnexion des réseaux de transport à haute tension du pool électrique d'Afrique Centrale (PEEAC). Or, l'intégration du projet CAB dans ces projets aurait permis - grâce à l'application de la méthodologie SIR - non seulement des économies importantes sur la réalisation des artères du réseau $C A B^{50}$, mais aussi sur la viabilisation, la fiabilisation et la sécurisation de ces axes routiers, essentiels pour la sous-région. Sans compter que l'intégration des infrastructures - réalisée selon la méthodologie SIR - est en même temps un puissant outil d'intégration sous-régionale, priorité affichée du NEPAD et de I'UA. Au printemps 2010, au cours de réunions organisées à sa demande, le CESIR a présenté et argumenté toutes ces observations et ces propositions relatives au projet $C A B$ de même que le concept et la méthodologie SIR et leur application à ce projet aux principaux acteurs impliqués : la Banque mondiale, la Commission européenne (DG Développement), la Banque européenne d'investissement (BEI), et l'UIT. La BAD en a été informée in extenso par écrit, de même que la Communauté économique des États d'Afrique centrale (CEEAC). Le CESIR a attiré l'attention de ses interlocuteurs sur les tares rédhibitoires de ce projet dont les deux principales sont sa topologie problématique et son accès insuffisamment sécurisé aux câbles sous-marins.

\footnotetext{
${ }^{49}$ Comme le réseau national à fibres optiques du Cameroun : $3200 \mathrm{~km}$ d'artères programmées. Une partie de ce réseau pourrait être utilisée comme artère (mixte, nationale et régionale)) du réseau $\mathrm{CAB}$. II faut savoir que le coût moyen de réalisation d'un $\mathrm{km}$ de câble à fibres optiques est voisin de, ou supérieur à vingt mille dollars en Afrique subsaharienne.

${ }^{50}$ À titre d'exemple, la seule intégration du projet $\mathrm{CAB}$ dans le projet de Corridor $\mathrm{n}{ }^{\circ} 1$ aurait permis d'économiser près de 10 millions de dollars pour la pose du câble à fibres optiques du $C A B$. Cette proposition avait été soumise aux acteurs intéressés(BM, BAD, BEI, UIT) lors de réunions à la demande du CESIR au printemps 2010 mais n'a eu aucune suite
} 
Deux ans après, rien n'a été entrepris et ce projet estimé à $215 \mathrm{M} \$$ par la Banque mondiale ${ }^{51}$ est ainsi bien parti pour figurer dans la déjà trop longue liste des "éléphants blancs ». Conclusion: ces institutions ne se considèrent non seulement infaillibles, mais elles sont aussi inébranlables. En outre, elles n'ont rien retenu de l'échec d'Africa ONE puisque qu'elles prescrivent « un partenariat public-privé (PPP) pour assurer la gestion de l'infrastructure de CAB »!

On assiste ainsi à une prolifération de projets et de réalisations d'artères à fibres optiques à travers le continent, sans cohérence et dont une partie est en doublon, sans concept de réseau résilient, et sans schéma intégrateur en vue d'une gestion rationnelle du trafic.

Or, l'initiative "Connect Africa », lancée par I'UIT en 2007 , avait comme principal thème le déploiement des TIC à travers le continent et s'était fixé comme objectif la réalisation d'un réseau continental africain à l'horizon 2012. Cet engagement s'est singulièrement dissolu dans l'obsession néolibérale de I'UIT qui se félicite systématiquement de chaque nouvelle réalisation nonobstant qu'elle participe de cette prolifération anarchique et sans cohérence d'artères et de réseaux. On est décidément bięn loin de Panaftel et de ce réseau terrestre continental qui était son ambition ${ }^{52}$ ! Comme on le verra ci-après, les artères sous-marines n'échappent pas à ce triste constat.

\subsection{Câbles sous-marins, ou enfin SAT-3 vint !}

L'histoire commence en 1996 lorsqu'un groupe d'opérateurs nationaux africains et européens décide-d'élaborer un projet de desserte, par câble sousmarin, des pays riverains de la côte ouest-africaine à partir de l'Europe (Sesimbra, Portugal). Ce câble devant succéder au câble SAT-2 ${ }^{53}$, existant

\footnotetext{
${ }^{51}$ Voir Press Release ${ }^{\circ}$ 2010/94.SDN de la Banque mondiale, Genève, 9 octobre 2009 - Titre : " A \$215M Central African Backbone Program Will Bring Low Cost, High Speed Internet to the Region" Texte : "Today the Executive Board of Directors of the World Bank Group has announced its endorsement of the $\$ 215 \mathrm{M}$, ten-year Central African Backbone Program (CAB Program). [...] 52

Lors de la session de clôture du Forum 2011 du SMSI, CSDPTT avait interpellé le secrétaire général de I'UIT en lui demandant d'accorder la priorité à la mise en œuvre de ce réseau eu égard à l'urgence, et suggéré de l'appeler Panaftel 2 en hommage à cette grande aventure qu'était, pour I'UIT et pour l'Afrique, le projet Panaftel. Sans répondre sur le fond, le secrétaire général a ironisé sur le côté "passéiste" de la proposition, concluant que "Panaftel est dépassé alors que nous sommes en pleine mondialisation, et les technologies ont fait d'énormes progrès depuis ».

53 South Africa Telecommunication $n^{\circ} 2$, mis en œuvre en 1993 pour succéder au câble sous-marin coaxial SAT-1, saturé et obsolète.
} 
mais proche de la saturation, il a pris le nom de SAT-3/WASC (West African Submarine Cable). On se rappelle qu'à cette date plusieurs pays africains se sont déjà engagés - moyennant finances - pour avoir un accès au câble du projet Africa ONE. Sauf l'Afrique du Sud et pour cause : son opérateur national, Telkom SA, a été sceptique dès le début par rapport à ce projet et a préféré être meneur (avec France Télécom notamment) sur SAT-3/WASC, d'autant plus que ce câble était prolongé vers l'Océan indien ${ }^{2}$ à partir de Melkbosstrand, son site d'atterrissement terminal en République sud-africaine. À la différence d'Africa ONE, SAT-3/WASC repose sur un modèle économique et financier traditionnel, les opérateurs nationaux parties prenantes finançant l'essentiel de l'investissement et suivant de près l'élaboration technologique du projet. Finalisé en 1998, SAT-3/WASC est attribué à Alcatel Submarine Networks suite à un appel d'offres international (son prolongement est attribué selon la même procédure à Tyco International). SAT-3/WASC est prêt fin 2001 et officiellęment inauguré le 26 mai 2002 à Dakar par le Président Abdoulaye Wade ${ }^{55}$. Le directeur général de Telkom South Africa (Telkom SA), Sizwe Nxasana, a pu déclarer : " [...] Je ressens un énorme sentiment de fierté dans la population d'Afrique. Ce système est une preuve tangible de notre détermination collective d'utiliser les synergies africaines pour trouver des solutions africaines à des défis africains [...] par cette infrastructure de communications de classe mondiale que nous possédons, contrôlons et maintenons nous-mêmes ".

À tous points de vue, SAT-3/WASC s'est ainsi avéré comme l'anti-modèle d'Africa ONE et sa réalisation dans les délais impartis a sonné comme un avertissement à I'UIT et en particulier à son BDT, complètement absent du projet SAT-3!

En même temps, ce projet, rondement mené et respectueux de son budget annoncé, 638 millions de dollars, sonne la fin de l'aventure Africa ONE et prouve combien l'UIT s'est fourvoyée, voire compromise, dans cette regrettable aventure qui a sérieusement écorné son crédit auprès d'un grand nombre d'États africains. La façade atlantique de l'Afrique est dès lors connectée aux grands réseaux mondiaux sous-marins et continentaux, via 12 stations d'atterrissement, et dispose d'un débit initial de $120 \mathrm{Gbit} / \mathrm{s}$; celui-ci est porté à $340 \mathrm{Gbit} / \mathrm{s}$ en 2009. Les 12 opérateurs nationaux africains copropriétaires de SAT-3/WASC ont le monopole de l'accès à ce "portail " international en échange de leur investissement; cela provoquera par la suite des problèmes

\footnotetext{
54 Le segment SAFE (South Africa Far East) desservira Madagascar, Maurice, la Réunion, l'Inde et Singapour.

55

Le Sénégal, à l'instar de l'Afrique du Sud, n'avait pas rallié le projet Africa ONE.
} 
avec les nouveaux opérateurs privés qui estiment que ces dispositions "biaisent le jeu de la concurrence ». Argument bien connu et récurrent des partisans de la loi du marché comme seul moteur du déploiement des " nouvelles technologies de l'information et de la communication » (NTIC ou $\mathrm{TIC}$ ) comme on appelle les télécommunications au nouveau millénaire.

\subsection{Concurrence plutôt que cohérence}

La prolifération des réalisations d'artères $\mathrm{FO}$ relève de l'absence totale de toute cohérence, où la concurrence est encouragée par I'UIT au détriment de l'indispensable coordination et de vision d'ensemble, qui sont non seulement une exigence basique pour tout réseau, mais évitent aussi une dilapidation scandaleuse d'énormes ressources financières. Ainsi, on ne compte pas moins de six réseaux à fibres optiques au Kenya, alors que ce même pays abrite le plus grand et le plus dangereux bidonville d'Afrique avec près d'un million d'habitants. À cette situation choquante le gouvernement a ajouté un autre scandale en finançant à $40 \%$ et sur fonds propres ( ?) le câble sous-marin Teams (The East African Marine System), qui relie Mombasa à Fujairah aux Émirats arabes unis (EAU) avec des débouchés hypothétiques, alors que deux autres câbles sous-marins (EASSy et Seacom) ${ }^{56}$ desservant la côte estafricaine allaient à leur tour desservir Mombasa. Plus de 130 millions de dollars ont ainsi été dilapidés - dont près de la moitié par le gouvernement kenyan avec le consentement tacite de l'UIT.

\subsection{La politique des comptoirs}

Ces sociétés, engagées dans leur logique du marché et dont la plupart sont appuyées sur des montages financiers de circonstance, vont prouver leur capacité de réaction sur ce segment si important qui leur a échappé, avec le soutien de I'UIT et des institutions financières internationales, toujours les mêmes. Elles sont bientôt rejointes par le Nouveau partenariat pour le développement de l'Afrique, connu sous son acronyme anglais NEPAD, ce qui pourrait paraître paradoxal si on oubliait que ses co-fondateurs sont le très néolibéral Président Wade du Sénégal et l'opportuniste Président Thabo Mbeki d'Afrique du Sud.

Ils s'appuient sur le fait que les opérateurs membres de SAT-3 ont un monopole d'accès et facturent donc aux opérateurs concurrents leur «bande

\footnotetext{
${ }^{56}$ East African Submarine System et South East African Communications.
} 
passante» ${ }^{57}$ à des tarifs jugés uni sono trop élevés par ces derniers, surtout en comparaison avec ceux en vigueur dans les pays développés. En outre, la simple évocation de monopole détenu par un opérateur "national » est une antinomie pour les concurrents potentiels dans un monde régi par la loi du marché. L'affaire devient politique, voire idéologique. Paradoxalement, ils oublient la "bulle Internet » et les deux mille milliards de dollars évaporés en trois ans dans des projets démesurés, de même que l'échec d'Africa ONE.

Des sociétés et entités, constituées selon des critères souvent disparates, vont donc réutiliser la formule des néolibéraux d'avant la bulle : "Construisons l'infrastructure, ils viendront l'utiliser » (Let's build, They'll come). II ne leur restera alors plus qu'à encaisser les contributions des candidats à la « bande passante »! Des rendements financiers de $15 \%$ sont une valeur de seuil minimal dans l'évaluation de la faisabilité de ces infrastructures, et comme l'Afrique présente le plus fort potentiel de développement, en particulier dans les télécommunications et TIC, ces groupes se lancent dès 2003 à l'assaut du continent, chacun espérant récompenser ses actionnaires. Leur stratégie n'est guère innovante puisqu'ils optent pour une politique des comptoirs qu'ont inventé et utilisé les premiers colonisateurs de l'Afrique! Alors que la côte Est de l'Afrique est encore privée de toute desserte côtière à haut débit, les premiers projets sont appliqués à la desserte par câbles sous-marins de la côte Ouest - la côte atlantique - du continent. La présence de pays riverains gros producteurs d'hydrocarbures n'est pas étrangère à cette stratégie, qui permet en même temps de concurrencer SAT-3/WASC, ouvertement et de manière offensive au plein sens du terme. La côte Est ne sera investie que deux ans plus tard et le premier câble sous-marin la desservant, Seacom, est inauguré en juillet 2009, soit plus de sept ans après SAT-3/WASC.

\subsection{Côte Ouest : de la rareté à la profusion}

Les projets naissent rapidement dans des bureaux d'étude improvisés de sociétés souvent au nom ronflant: Infinity, Global-1, Main One, etc. La communication commerciale des protagonistes est centrée sur la baisse, drastique (dramatical), des tarifs des « bandes passantes » pour l'Internet et des

57 Cette expression, qui s'exprime en $\mathrm{Hz}, \mathrm{kHz}, \mathrm{MHz}, \mathrm{GHz}$, etc., est faussement utilisée en technologie numérique si on veut parler de "ressource » utilisée pour communiquer ou disponible pour la communication. En technologie numérique, cette ressource s'exprime en terme de débit binaire, donc en bit/s et en ses multiples : kbit/s, Mbit/s, Gbit/s, Tbit/s, etc. Dans la suite, nous parlerons ainsi de débit et mettrons entre parenthèses la terminologie impropre... mais largement utilisée dans les médias ! 
appels internationaux selon le principe, néolibéral et simpliste, que plus il y a d'opérateurs concurrents, moins la communication sera chère. Ainsi, l'agence de presse Bloomberg prévoit que l'arrivée d'un nouveau câble sous-marin en République Sud-Africaine pourrait faire chuter le prix du haut débit de $90 \%(58)$ alors que l'analyste Pyramid Research n'évalue ces baisses qu'à $5 \%$ par an ${ }^{58}$. D'autre part, on oublie que la multiplication d'artères de communication implique la multiplication des investissements... et la diminution des revenus par utilisateur pour chacun des opérateurs de ces artères ${ }^{59}$. Leurs revenus sont ainsi a minima et ne permettent plus de recourir à l'autofinancement - total ou au moins partiel - pour étendre et développer leur réseau. Pour ce faire, ils seront désormais liés à des financements extérieurs. Cette évolution est un véritable changement de paradigme dont I'UIT et les institutions financières internationales n'ont guère mesuré l'impact.

En l'espace de six ans, on verra ainsi naître une dizaine de projets, et les médias africains leur témoignent une complicité troublante. Ainsi, ils reprennent presque mot pour mot les communiqués et informations distillés par les "communicateurs" de ces sociétés ou groupes, et retransmettent à leurs concitoyens l'avenir radieux peuplé de services les plus sophistiqués au moindre coût, donc le "progrès ». En outre, on assiste à un glissement sémantique symptomatique: toute la «com» est centrée sur Internet, évidemment "à large bande " (broadband), car le "progrès » en Afrique ne saurait se compter en centaines de kbit/s mais en dizaines de Mbit/s ! Exunt donc la téléphonie, les services vocaux et autres échanges de données d'entreprises... C'est finalement la « crise des subprimes » de 2009 qui limitera les ambitions irrationnelles de ces sociétés, plus aventurières de la finance qu'opérateurs de réseau et prises à leur propre piège.

À cette date, on compte les câbles et projets suivants :

SAT-2 : Ce câble à fibres optiques de $9500 \mathrm{~km}$ avec 82 répéteurs a été mis en service en 1993. II va de l'île de Madère - où il est relié au système des câbles sous-marins européens - à Melkbosstrand en République Sud-Africaine et a une capacité installée de $560 \mathrm{Mbit} / \mathrm{s}$. Propriété de 5 grands opérateurs, dont

\footnotetext{
58 Voir Julien Clémençot, «Télécoms : une révolution sous-marine », Jeune Afrique, 19 septembre $2010, n^{\circ} 2593$

59 C'est le fameux ARPU que l'on trouve dans un grand nombre de publications: Average Revenue Per User, ou revenu moyen par utilisateur. Sauf indication contraire il est établi sur une base mensuelle.

60 Voir Jean-Louis Fullsack «Infrastructures de base pour le développement des médias électroniques en Afrique » in: Les médias de l'expression de la diversité culturelle en Afrique, sous la dir.de Théophile Balima et Michel Mathien, éditions Bruylant, 2012, p. 109-124
}

tic\&société - 5 (2-3), $2^{\text {ème }}$ semestre $2011-1^{\text {er }}$ semestre 2012 
le sud-africain Telkom, il a remplacé le câble SAT-1 (coaxial, 360 voies) posé dans les années 1960. II assure aussi la sécurisation partielle du trafic de SAT-3 en cas de panne de celui-ci.

SAT-3 : Ce câble sous-marin, qui dessert la côte atlantique de l'Afrique en 9 stations d'atterrissement (une dixième vient d'y être ajoutée), est en exploitation sous forme coopérative depuis mai 2002. Long de $14300 \mathrm{~km}$, il a une capacité de $120 \mathrm{Gbit} / \mathrm{s}$ (4 fibres, amplification optique, multiplexage en longueurs d'onde) à sa mise en service et a coûté $638 \mathrm{M} \$$. Selon les analystes, il est utilisé à moins de $20 \%$ de sa capacité installée et ses coûts d'accès sont trop élevés : $4000 \$$ et plus par Mbit/mois, ceci expliquant la réaction des opérateurs non membres du consortium... et justifiant l'émergence d'une concurrence dans une économie de marché. En 2011, SAT-3/WASC reste cependant l'artère de desserte principale de la côte ouest-africaine.

Infinity : Ce câble est conçu et projeté en 2006 par la société américaine IWTG . II doit s'étendre du Portugal au Cameroun et être opérationnel en 2008... Son parcours est strictement « en doublon » de SAT-3 dont il ambitionne de "récupérer " une bonne part de trafic grâce à une offre dite d'accès ouvert (open access) " significativement moins chère " que SAT-3. Long de 7000 km et avec une capacité " supérieure à 1 Térabit/s », son coût annoncé varie entre 600 et $865 \mathrm{M} \$$. Son offre de connectivité minimum est de $10 \mathrm{Gbit} / \mathrm{s}$ par pays. Ce projet entièrement privé ${ }^{62}$, avec pour but ouvertement affiché de concurrencer SAT-3, repose donc essentiellement sur des bases idéologiques... mais ne s'est jusqu'à présent (mars 2012) pas réveillé de ce repos. En effet, en 2011, Infinity a disparu des écrans, victime de la "nouvelle économie "; au moins a-t-on économisé plus de huit cent $\mathrm{M} \$$...

Main One : Ce câble entièrement privé et détenu par des Africains est conçu sur le modèle de "réseau ouvert » : sa société vend " en gros" de la capacité à tout opérateur ou prestataire de services qui le lui demande. II relie le Portugal (Seixal) à l'Afrique du Sud... comme SAT-3, mais avec moins de sites d'atterrissement. II est programmé sur deux phases. La première relie Setubal à Lagos (Nigeria) en $7200 \mathrm{~km}$ et une capacité potentielle de 1,92 Tbit/s pour un coût annoncé de $250 \mathrm{M} \$$, probablement sous-évalué. En 2008, il n'a que deux licences d'atterrissement, au Ghana et au Nigeria, alors que son exploitation était prévue pour juin 2010. Sa réalisation a été confiée à Tyco qui en fournit

\footnotetext{
61 Infinity Worldwide Telecommunications Group.

62 M. Yaya Kourouma, chef de la Division Afrique du BDT-UIT et personne contact de I'UIT pour le projet Africa ONE jusqu'en 2001, est conseiller du Directoire du projet Infinity.
} 
aussi l'équipement. Sa connectivité limitée pose deux questions : (a) pourquoi une telle capacité pour si peu de connectivité, et (b) quelle viabilité pour un tel projet ? D'autant qu'il a (déjà) un autre concurrent, Glo-1. En 2011, seule la phase I a été réalisée, et seuls le Ghana et le Nigeria sont desservis. Un échec au moins partiel, car sa capacité - tellement vantée par rapport à SAT-3/WASC est très largement sous-utilisée. Cela n'empêche pas ses responsables de vouloir réaliser la prolongation du câble sous-marin jusqu'en Afrique du Sud et de prévoir sa mise en service pour la fin de l'année $2012^{63}$. II est en partenariat avec Seacom (côte Est de l'Afrique) et cherche un autre partenaire pour.... "ceinturer le continent ». Africa ONE : le retour?

Glo-1 : Ce câble doit relier le Royaume-Uni au Nigeria (Lagos) et comporter " quelques embranchements " sur la côte Ouest africaine. Outre le Ghana et le Nigeria, le Sénégal et la Gambie ont signé une licence pour le site d'atterrissement. Réalisé par Alcatel, en principe à partir de 2005, il n'arrive à Lagos qu'en septembre 2009. Son coût prévu est de $250 \mathrm{M} \$$ pour son segment jusqu'à Lagos, et pour une capacité finale de 2,5 Tbit/s. Mais ce projet - qui bénéficie du soutien du NEPAD - est ambitieux puisqu'il envisage de connecter I'Afrique à l'Inde, au Moyen-Orient, au Royaume-Uni et aux États-Unis. Le projet comporte aussi un réseau de liaisons terrestres, complémentaires des liaisons sous-marines, ce qui met son coût final à hauteur de 1,5 milliard de dollars. Ses protagonistes prévoyaient son achèvement en 2010. En 2011, il compte des licences pour la Côte d'lvoire et le Bénin et met en service sa station d'atterrissement à Accra, tout en annonçant qu'il reliera 14 pays africains à l'Europe, avec une liaison avec les États-Unis. Avec $30 \mathrm{Gbit} / \mathrm{s}$ annoncés en septembre $2009^{64}$, il est notoirement sous-utilisé si tant est qu'il est en exploitation réelle ! Au mieux, les centaines de millions de dollars investis dorment depuis des années...

WACS (West African Cable System), aussi appelé AWCC (African West Coast Cable System), est porté par un consortium rassemblant onze opérateurs conduit par Broadband Infraco ${ }^{65}$, une société d'économie mixte sud-africaine.

\footnotetext{
63 Rudolph Muller, "Main One's South African plans», My BroadBand, 15 July 2010 www.mybroadband.co.za/news/

64

Balancing Act News Update, September 2009, Issue $\mathrm{n}{ }^{\circ} 473$

http://www.balancingact-africa.com/news/archive/english

${ }^{65}$ On retrouve un projet de câble sous-marin ouest-africain sous le nom d'Infraco dans certaines sources (notamment l'agence Terabit Consulting). En fait, Infraco est un opérateur d'opérateurs (carriers' carrier) appartenant au gouvernement sud-africain, dont la fonction de base est la fourniture de "bande passante " à des opérateurs ou entreprises privés, mais qui connaît de graves et coûteuses difficultés.
} 
Selon ses promoteurs, très discrets sur son coût estimatif qui pourrait dépasser $650 \mathrm{M} \$$, il aura une capacité de 3,8 Tbit/s (extensible à 5,12 Tbit/s) et doit desservir dix pays côtiers et le Cap Vert. D'une longueur totale de $14900 \mathrm{~km}$, il atteindra Lagos au début de 2011 ; le 19 avril 2011, il atteint la nouvelle station d'atterrissement d'Yzerfontain en Afrique du Sud ${ }^{66}$. L'entrée en service du câble est prévue pour le premier trimestre de 2012 avec une capacité initiale de 500 Gbit/s.

ACE (Africa Coast to Europe) est un câble sous-marin entre la France et l'Afrique du Sud conçu et géré par un consortium emmené par France Télécom et composé de 25 opérateurs (dont six filiales africaines d'Orange). Long de 17 $000 \mathrm{~km}$, il a une capacité minimale de 1,92 Tbit/s et doit desservir une vingtaine de pays côtiers africains en deux phases, la première réalisant le segment France-Gabon. Son coût prévisionnel est de $700 \mathrm{M} \$$. Cependant, le projet ACE est intégré dans le projet de réseau sous-marin du NEPAD connu sous le vocable Uhurunet ${ }^{67}$ après l'accord signé en juin 2010 avec France Télécom par Baharicom Development Company (BDC), la société holding de Uhurunet, qui devient ainsi co-coordinateur du projet ACE avec France Télécom. Cet accord aboutira à la réalisation en une traite de l'ensemble de ce projet qui devait s'achever à la fin 2011. On trouvera donc deux appellations pour ce câble : ACE et ACE/Uhurunet.

WAFS (West African Festoon System) est un projet original qui devait aller de Lomé (Togo) à Luanda (Angola), soit plus de $2000 \mathrm{~km}$ en 10 festons (segments sous-marins entre deux sites d'atterrissement) et cinq dérivations (dont Malabo - Guinée Equatoriale). L'idée est intéressante si les festons sont purement passifs (pas de répéteurs immergés), car l'exploitation et le développement (augmentation de capacité) sont entièrement maîtrisables par les pays desservis, et les coûts d'exploitation seraient significativement réduits. Cependant, le projet ne semble guère avoir dépassé le stade du « Powerpoint Network »... Dommage, car c'est précisément ce type de liaisons qui devrait intéresser en priorité les responsables africains.

MT WAC (Maroc Telecom West Africa Cable) est un projet de câbles sousmarins s'étendant du Portugal au Gabon, avec des festons desservant le Maroc, le «Sahara occidental » (!), la Mauritanie, le Sénégal et la Côte d'Ivoire. II est conçu essentiellement pour collecter le trafic des filiales de MT/Vivendi de la

\footnotetext{
66 L'Afrique du Sud possède désormais trois stations d'atterrissement : Melkbosstrand, Mtunzini et Yzerfontain.

${ }^{67}$ La partie terrestre du projet de réseau continental africain est appelé Umojanet.
} 
Jean-Louis FULLSACK

sous-région, et l'appel d'offres devait être lancé en 2009 "au plus tard». En 2011 il n'y a aucune trace de ce projet.

SAT-4 : OUI déjà ! C'est le successeur de SAT-3. II est dans les cartons de Telkom, Vodacom et MTN, les trois «majors" sud africains des télécommunications. On pense qu'il pourrait « récupérer» tout ou partie de AWWC. À suivre... 
La gouvernance discutable de I'UIT : Le projet Africa ONE comme exemple

Tableau des systèmes sous-marins de la côte ouest-africaine (état en mai 2011)

(les coûts sont ceux annoncés par les concepteurs ou réalisateurs des projets)

\begin{tabular}{|c|c|c|c|c|c|c|c|}
\hline Système SM & Réalisé & Programmé & Planifié & Envisagé & Date & $\begin{array}{l}\text { Coût } \\
\text { en M\$ }\end{array}$ & Renvoi \\
\hline SAT-2 & $\mathbf{x}$ & & & & 1993 &.$/$. & \\
\hline SAT-3/WASC & $\mathbf{x}$ & & & & 2001 & 638 & \\
\hline Main One & $x$ & & & & 2010 & $250+$ & Phase 1 \\
\hline Glo-1 & $\mathbf{x}$ & & & & 2009 & $250+$ & Phase 1 \\
\hline WACS & & $\mathbf{x}$ & & & 2012 & $650+$ & \\
\hline$A C E$ & & $x$ & & & 2012 & 700 & \\
\hline Infinity & & & $X ?$ & & $?$ & $800+$ & En attente \\
\hline WAFS & & & & $\mathbf{x}$ & $?$ & $?$ & \\
\hline MT WAC & & & & $\mathbf{x}$ & $?$ & & \\
\hline$S A T-4$ & & & & $\mathbf{x}$ & $>2012$ & & \\
\hline $\begin{array}{l}\text { Coût total } \\
\text { investi }\end{array}$ & & & & & & $2388++$ & Hors Infinity \\
\hline
\end{tabular}

Cette foison de projets crée une situation difficilement acceptable pour au moins deux raisons.

Sur le plan technologique, elle montre l'absence totale d'un véritable concept de réseau, et cela à deux niveaux : 1- Au niveau des stations d'atterrissement et du câble sous-marin, les différents systèmes sont gérés comme des entités indépendantes et n'offrent par conséquent aucune redondance entre eux. Dès lors, une défaillance totale d'une station d'atterrissement (par exemple une coupure de son alimentation électrique) interrompt tout le trafic acheminé par l'ensemble des câbles sousmarins qu'elle accueille. Une défaillance partielle affectant une ou plusieurs terminaisons de câbles sous-marins interrompt le trafic acheminé par le/s 
câble/s incriminé/s. Dans ces deux cas, le trafic international d'un vaste arrièrepays concentré par la station d'atterrissement est respectivement complètement ou partiellement interrompu.

2 - Au niveau des sous-régions ${ }^{68}$ que desservent les stations d'atterrissement, il n'existe aucun plan de routage de secours en cas de rupture de l'une des grandes artères d'infrastructure; dès lors, une coupure d'artère continentale coupe aussi l'accès de tout un arrière-pays au câble sous-marin.

L'absence d'une architecture de sécurisation mutuelle à ces deux niveaux de connectivité des câbles sous-marins a donné lieu à de nombreuses et longues interruptions totales du trafic et, par conséquent, à des pertes importantes de recettes d'exploitation pour les opérateurs, auxquelles il faut ajouter le manque à gagner des entreprises et acteurs économiques dans de vastes zones privés de communications.

À cette carence s'ajoute l'absence d'idée directrice quant à la gestion, l'exploitation et le développement de cet écheveau de câbles sous-marins. C'est la conséquence inéluctable du principe de la concurrence incontrôlée, incompatible par nature avec la mise en place d'une structure permettant la mutualisation des ressources d'accès et leur évolution cohérente en fonction des besoins en terme de débit. Une fois de plus, c'est le concept néolibéral qui prévaut : la concurrence plutôt que la cohérence

C'est la première raison de cette situation difficilement acceptable et elle met particulièrement en cause les organisations internationales : I'UIT pour ce qui concerne l'intégration de l'ensemble des systèmes sous-marins en un concept de réseau résilient, la $B M$ et la BAD qui co-financent ces projets, et I'Union africaine qui n'exerce pas sa fonction de souveraineté quant à la finalité de ces réalisations. Au lieu de cela, ces mêmes acteurs soutiennent passivement - s'ils ne l'encouragent pas - la promotion (et le financement), la prolifération anarchique et en grande partie inutile ${ }^{6}$ (voir les capacités réelles utilisées par rapport au potentiel installé) d'artères de télécommunications en Afrique.

${ }^{68}$ Pour l'Afrique sub-saharienne et dans la problématique discutée, ce sont les sous-régions ayant une façade atlantique, soit les communautés économiques d'Afrique de l'Ouest (CEDEAO), d'Afrique Centrale (CEEAC) et d'Afrique du Sud (SADC).

${ }^{69}$ La plupart des câbles sous-marins africains sont peu à très peu chargés (moins de $15 \%$ de leur capacité) ; c'est probablement la raison pour laquelle on ne trouve aucune donnée exacte de leur capacité utilisée et/ou du rapport entre cette capacité et la capacité installée, et encore moins entre la capacité utilisée et la capacité potentielle du câble (celle que permettent les caractéristiques intrinsèques des fibres et des équipements des sections immergées).

tic\&société - 5 (2-3), $2^{\text {ème }}$ semestre $2011-1^{\text {er }}$ semestre 2012 
Sur le plan financier, cette multiplicité de projets et de réalisations constitue un autre thème critique. Ainsi l'étude de Terabit Consulting (www.terabitconsulting.com) chiffre l'ensemble des projets sous-marins, côtes Ouest et Est et hors projet ACE, à 6,1 milliards de dollars soit dix fois le coût de SAT-3, alors que les coûts dans ce domaine ont diminué de $30 \%$ depuis sa mise en service. Si l'on ne considère que la côte Ouest-africaine, deux ou trois systèmes sous-marins parmi les six qui seront installés en 2012 suffiraient amplement pour assurer le trafic international pendant la décennie à venir (sans compter que quatre autres sont encore dans les cartons...) ; on déduit que plus de 2 milliards de dollars auront été dépensés dans des investissements inutiles, dans une période de crise financière dont la population africaine est la première victime, jusque dans ses besoins les plus primaires. Ce véritable détournement de fonds constitue la deuxième raison d'une situation rationnellement et humainement inacceptable.

L'attitude irresponsable, voire cynique, des acteurs concernés, les organismes pan-africains comme le NEPAD et les responsables nationaux, mais aussi I'UIT qui a complètement déserté son champ d'activité fondamental pour se focaliser sur la déréglementation des opérateurs et promouvoir l'économie de marché au détriment de la coopération, indispensable - à condition d'être bien ciblée - pour les pays africains. Leur comportement ignorant à ce point les principes élémentaires de dignité et de responsabilité est, pour toutes les raisons évoquées plus haut, unanimement condamnable et constitue un troisième champ de critique.

\section{Conclusion}

Le projet Africa ONE a été plus qu'un indicateur de la dérive néolibérale de I'UIT. II a aussi parfaitement illustré deux tares fondamentales de cette agence onusienne: le refus d'assumer ses responsabilités et l'abandon de sa compétence technologique, en particulier celle relative à la conception, au déploiement et au développement des réseaux dans les PeD. En outre, son assistance en expertise auprès des institutions internationales de financement (BM et $\mathrm{BAD}$ notamment) a montré une insuffisance technologique notoire à l'exemple de son implication dans le projet $C A B$ en Afrique Centrale. Enfin, ajoutant à ses graves lacunes citées son manque de réactivité, I'UIT est en grande partie responsable de l'absence d'un réseau d'interconnexion continental pour l'Afrique, Panaftel, qu'elle s'était pourtant engagée à réaliser pour l'an 2000 lors de la création de l'Union africaine. 
Jean-Louis FULLSACK

Les dérives et erreurs stratégiques de I'UIT relevées précédemment appellent au retour de cette agence sur ses fondamentaux comme CSDPTT l'a demandé tout au long du SMSI. De même, I'UIT devra reconquérir ses compétences, voire sa maîtrise, en matière de réseaux et d'architectures résilientes, et plus généralement dans les technologies de l'information et de la communication (TIC) adaptées aux besoins et aux possibilités des pays en développement, afin d'offrir à ses États membres - en premier lieu les PeD - le concours efficace et la coopération authentique dont ils ont un urgent besoin. C'est donc un véritable aggiornamento, voire une révolution culturelle, qu'il faudra imposer au BDT.

En outre, - a fortiori comme coordonnatrice du SMSI qu'elle proclame multipartenarial -, I'UIT doit impérativement s'ouvrir aux organisations de la société civile dont l'activité et les objectifs relèvent du domaine de son BDT, comme le demande CSDPTT depuis le début du SMSI. Une telle ouverture l'enrichirait par un apport supplémentaire de compétences, et contribuerait à un relatif rééquilibrage de cette agence onusienne où le secteur privé a pris une place et une influepce surdimensionnées qui ont joué un rôle primordial dans sa dérive néolibérale .

Enfin, I'UIT devra redevenir le partenaire fiable et compétent de ses États membres. À cette fin, elle devra abandonner certaines relations discutables, telles que le partenariat avec la société Cisco pour le déploiement d' " académies Cisco ", et renouer avec une collaboration, directe et soutenue, des établissements d'enseignement supérieur technique des PeD, notamment en Afrique, afin de donner aux Africains les connaissances et les moyens pour résoudre plus largement leurs problèmes et non ceux spécifiques à Cisco.

Ce retour aux fondamentaux de I'UIT ne se fera pas par simple incantation ou invocation des réalités, mais nécessite une mobilisation des organisations de

\footnotetext{
70 À propos de cette dérive et de ces excès, on lira avec intérêt l'article d'Antonio Pasquali, ancien directeur général de l'Unesco, secteur de la Communication, intitulé «La "société de l'information" : des précédents qui plaident pour la mise en place d'un Tribunal International » (voir www.moviementos.org/foro_communicacion) 71

Ce partenariat signé en 2000 est alimenté par une contribution de 12 millions de dollars de I'UIT dont le budget est alors en déficit - et de 8 millions de dollars pour Cisco, quasi monopoliste des routeurs de réseau qui a fait 8 milliards de bénéfices annuels! L'UIT offre ainsi le marché en extension des $\mathrm{PeD}$ et un vivier de techniciens et ingénieurs " low cost " à l'équipementier américain tout en privant les établissements d'enseignement technique supérieur des PeD de leurs meilleurs éléments, enseignants comme étudiants. Voir Jean-Louis Fullsack, " L'UIT acteur déterminant dans l'évolution néolibérale du secteur des télécommunications ", in: Les télécommunications entre bien public et marchandise, p. 347-364, op.cit. et le site aspd.revues.org/558, Hamadoun Touré "Plus que la fracture, c'est l'opportunité numérique qui importe », propos recueillis par Michel Egger.
}

tic\&société - 5 (2-3), $2^{\text {ème }}$ semestre $2011-1^{\text {er }}$ semestre 2012 
la société civile et des citoyens au "Nord " comme au "Sud", afin que les responsables politiques prennent le relais et recentrent non seulement I'UIT mais tout le système des Nations unies sur les fonctions et devoirs inscrits dans leurs bases fondatrices. Cet objectif ne sera atteint que si le milieu universitaire en mettant en œuvre ses ressources en études et en recherche - s'implique délibérément dans ce vaste mouvement et éclaire les acteurs, citoyens et politiques, sur les problématiques fondamentales créées par ces agences onusiennes et par leurs dérives néolibérales. II contribuera ainsi à cette mobilisation et permettra aux forces vives de la société civile d'identifier et de proposer des voies de sortie de ce modèle néolibéral qui a fait son temps et beaucoup trop de dégâts. 